\title{
Hilar and Mediastinal Involvement in Bronchogenic Carcinoma: CT Evaluation and Histopathologic Correlation
}

\author{
${ }^{1}$ Sayed Jamiul Hossain, ${ }^{2}$ Md. Rakibul Hassan, ${ }^{2}$ Md. Shafiqur Rahman, ${ }^{3}$ Md. Abu Baker Siddique and ${ }^{4}$ Md. Sahajahan \\ ${ }^{1}$ Senior Medical Officer, Institute of Nuclear Medicine and Allied Sciences, Cox's Bazar \\ 2 Associate Professor. Dept. of Radiology and imaging, JIMCH \\ ${ }^{3}$ Senior Medical Officer, Institute of Nuclear Medicine and Allied Sciences Mitford, \\ ${ }^{4}$ Assistant Professor, Dept. of Radiology and imaging, East-West Medical College, Hospital.
}

Correspondence Address : Sayed Jamiul Hossain, Senior Medical Officer, Institute of Nuclear Medicine and Allied Sciences, Cox's

Bazar, Email : dr.jamiul@gmail.com

\begin{abstract}
This cross sectional study was done to assess the diagnostic accuracy of Computed Tomography (CT) in the evaluation of hilar and mediastinal involvement in bronchogenic carcinoma. A total of 104 patients with bronchial mass lesion were included in the study. Thorough history, clinical examinations, X-ray chest, CT chest and CT guided fine needle aspiration cytology (FNAC) were done. This study was carried out in the department of Radiology and Imaging of Bangabandhu Sheikh Mujib Medical University (BSMMU) and Dhaka Medical College Hospital (DMCH) from July 2009 to June 2010. Diagnosis of hilar and mediastinal involvement in bronchogenic carcinoma by CT scan was highly sensitive and specific. CT findings of the present study correlated well in most of the cases with the CT guided FNAC results. It can therefore be concluded that CT scan is a useful modality in the evaluation of hilar and mediastinal involvement in bronchogenic carcinoma.
\end{abstract}

Keywords : Hilar and mediastinal involvement, bronchogenic carcinoma, CT evaluation

\section{INTRODUCTION}

Lung cancer is the most common cause of death in both men and women in the United States (US). It kills over one million people a year in whole world. Extensive prospective epidemiologic data clearly established cigarette smoking as the major cause of lung cancer. It is estimated that about $90 \%$ of male lung cancer deaths and $75-80 \%$ of lung cancer deaths in the US are caused by smoking each year (1). Clearly, lung cancer is an important and widespread disease that constitutes a major public health problem.

Ninety percent (90\%) of patients with lung cancer of all histological types are current or former cigarette smokers. Several established or suspected human carcinogens are present in the work environment and it is estimated that $3 \%$ to $17 \%$ of lung cancers are occupationally related (2).

Chest CT is the modality of choice for evaluating bronchogenic carcinoma because of its high spatial resolution. CT provides precise characterization of size, contour, extent and tissue composition of suspicious lesion. Mediastinal lymph nodes are better evaluated by CT than chest X-ray. CT is now established as the principal radiographic adjunct to $\mathrm{X}$-ray chest examination in the diagnosis and management of lung cancer. It should be used in the evaluation of every pulmonary nodule to determine whether the nodule is solitary and whether mediastinal metastases are present and to evaluate the mass by assessing its density. In general, nodules with Hounsfield numbers greater than +175 can be presumed to be calcified and, hence, benign. CT is of great value in determining the extent of lung cancer and at present the best imaging modality for evaluating mediastinal lymph nodes. Spread of tumour to mediastinal nodes is evaluated on the basis of node size. Nodes less than $1.0 \mathrm{~cm}$ in diameter are considered normal, $1.0-1.5 \mathrm{~cm}$ suspicious for tumour, and greater than $1.5 \mathrm{~cm}$ have a high probability of being malignant. Node size, however, is dependent on location in the mediastinum and whether infection is present in the lung. Size criteria alone should not be used to refuse surgery (3). 
With widely accepted spiral CT and newly developed multi-detector row CT techniques, the sensitivity of detecting pulmonary nodules has further improved (4). Recently, spiral CT technique has been applied to screening for lung cancer in high risk populations and shown to be useful in detecting early lung cancers (5). High-resolution, three-dimensional imaging of the thorax can allow us to evaluate small nodules at early stages. With sequential follow-up scans, changes in nodule number and size can be assessed. If CT for lung cancer screening becomes widely practiced, we expect that the early diagnosis of bronchogenic carcinoma will be dramatically rise and significantly reduce the death rate from this potential malignancy. In addition, computer-aided diagnosis (CAD) systems can help to improve a radiologist's performance for detecting pulmonary nodules (6).

General objective of this study was to assess the diagnostic accuracy of Computed tomography (CT) in evaluation of hilar and mediastinal involvement in bronchogenic carcinoma. Specific objectives were to diagnose and characterize the different types of bronchogenic carcinoma by CT scan, to correlate the CT findings of bronchogenic carcinoma with that of the cytopathological findings and to determine and validate the diagnostic sensitivity, specificity and accuracy of CT scan in diagnosing bronchogenic carcinoma.

\section{MATERIALS \& METHODS}

This cross sectional study was carried out on 104 patients with bronchial mass lesion in Radiology and Imaging Department of BSMMU and DMCH from July 2009 to June 2010 to confirm the clinical diagnosis by comparing $\mathrm{CT}$ scan with cytopathological findings. The patients were selected conveniently and purposively. Prior to the commencement of this study, the research protocol was approved by the ethical committee of BSMMU. The aims and objectives of the study along with its procedure, alternative diagnostic methods, risks and benefits of this study were explained to the patients in easily understandable local language and then informed written consent was taken from each patient. Thorough history, particularly symptoms related to bronchial mass lesion is carefully elicited to obtain maximum possible information regarding the illness. Diagnosis was established by the combination of history, physical examination, laboratory and x Ray chest. Then al patients ( $\mathrm{n}=104)$ underwent $\mathrm{CT}$ examination of lung. At both BSMMU and DMCH, CT was done by SIEMENS SOMATOMS EMOTIO DUO machines and $2-10 \mathrm{~mm}$ thick contiguous slice were taken. These scan were obtained with $120 \mathrm{kv}$ and $75 \mathrm{ma}$. Both pre and port contrast scan were performed. Non-ionic water soluble iodinated contrast medium (Iopamidol 375) was administered as bolus dose by medium hand pressure (at a rate of about 1-1.5 ml/sec with 19 gauze needle ) and completed within 50-60sec. Immediately after completion of bolus injection CT scan of chest were obtained including upper abdomen (targeting both adrenal glands). CT guided FNAC was performed in 102 patients as two patients were unfit for FNAC and cytopathology reports were not available in two patients. All CT scans were interpreted by the investigator initially and then were reviewed by the senior Radiologists of the Department Radiology and Imaging of BSMMU and DMCH. Finally CT findings of 100 patients were compared with their cytopathological reports.

The data were analyzed with the help of SPSS-13 (Statistical package for social sciences) software programmed. For the validity of the study outcome sensitivity, specificity, accuracy, positive and negative predictive values were calculated out according to the formulae described by Knapp and Miller (1992) (7).

\section{RESULTS}

Initially a total of 104 cases were selected who attended the departments of Radiology and Imaging, of BSMMU and DMCH with bronchial mass lesion 
were selected for this study. CT scan of chest was done in each of the patient. CT guided FNAC could not be done in 2 patients due to illness. Cytopathological examinations were done from respective pathology departments in 102 patients but two reports area not available. So, finally four patients were excluded from this comparative study. CT findings of 100 patients were compared with the cytopathological findings.

\section{CT scan findings of 104 patients with bronchial} mass lesion:

Out of 104 patients, left lung involvement was in 18 patients (17.3\%), right lung in 42 patients (40.4\%) and both lung in 44 patients $(42.3 \%)$. Apical segment was involved in $32(30.8 \%)$, anterior segment in 40 (38.5\%), posterior segment in $24(23.1 \%)$, posterior basal in $4(3.8 \%)$, and lateral segment in $4(3.8 \%)$ patients. Single involvement was found in $39(37.5 \%)$ and multiple involvements in $65(62.5 \%)$ patients $(\mathrm{n}=104)$. Out of 104 patients $57(54.8 \%)$ lesions were speculated and 47 (45.2\%) were smooth.

CT findings of hilar enlargement was $53.8 \%$, cavitation $26 \%$, rib destruction $37.5 \%$, elevation of hemi diaphragm $33.7 \%$, mediastinal involvement $14.4 \%$, chest wall invasion $17.3 \%$, air bronchogram $7.7 \%$, pleural effusion $58.7 \%$ and contrast enhancement $59.6 \%$, Out of 104 patients mild contrast enhancement is $50 \%$, moderate enhancement $50 \%$ and intense enhancement $4 \%$. Homogeneous enhancement was found in $34 \%$ and heterogeneous in enhancement $70 \%$ cases.

Among 104 patients of the study group the most common diagnosis was malignant lesion in 64 patients $(44.2 \%)$ followed by pulmonary tuberculosis in 20 patients $(19.2 \%)$, inflammatory lesion 14 patients (13.5\%), pneumonic consolidation in 4 patients $(3.5 \%)$ and fungal lesion in 2 patients (1.9\%). All 64 patients with suspected malignant tumor underwent FNAC but among 40 benign lesions FNAC could not be done in 2 patients and in 2 patients cytopathology reports were not available.

\section{Cytopathological diagnosis:}

In this study, among 100 patients, cytopathology confirmed malignancy in 62 (62\%) cases and other benign conditions in $38(38 \%)$ cases. The most common lung malignancy was squamous cell carcinoma. Twenty nine patients $(46.8 \%)$ were diagnosed cytopathologically as squamous cell carcinoma. Adenocarcinoma was diagnosed in 23 patients $(37.1 \%)$, small cell carcinoma in 6 patients $(9.7 \%)$ and large cell carcinoma in $4(0.7 \%)$ patients. Out of 38 benign condition cytopathology confirmed pulmonary TB in 19 patients (50\%), benign bronchial lesions in 7 patients (18.4\%), nonspecific inflammatory lesions in $8(21.1 \%)$, and pneumonic consolidation 4 patients (10.5\%).

Comparison between CT findings and cytopathological findings of lung masses.

Out of 100 patients who had both CT scan and FNAC, CT scan detected lung tumors in 64 and other benign condition in 36 patients. Out of 64 patients cytopathology conformed malignancy in 61 (True positive) and benign in 3 patients (false positive). Rest 36 cases diagnosed as benign conditions by CT, cytopathology confirmed bronchogenic carcinoma in 1 (false negative) and benign conditions in 35 patients (true negative). Table 1 shows the comparison between CT findings and cytopathological findings $(\mathrm{n}=100)$.

Table 1: Comparison between CT scan and cytopathological findings

\begin{tabular}{llll}
\hline \multirow{2}{*}{ CT diagnosis } & \multicolumn{2}{l}{ Cytopathological diagnosis } & \multirow{2}{*}{ Total } \\
\cline { 2 - 3 } & Positive & Negative & \\
\hline Lung Tumor & $61(\mathrm{TP})$ & 3 (FP) & 64 \\
Others & $1(\mathrm{FN})$ & $35(\mathrm{TN})$ & 36 \\
Total & 62 & 38 & 100 \\
\hline
\end{tabular}

Sensitivity of CT to diagnose lung tumors was $98.3 \%$, specificity $92.1 \%$, positive predictive value (PPV) $95.3 \%$, negative predictive value (NPV) $97.2 \%$ and accuracy $96.0 \%$. Table 2 shows the result of Validity tests. 
Table 2: Statistical analysis of the CT in the diagnosis of bronchial mass lesion.

\begin{tabular}{ll}
\hline Validity test & Percentage \\
\hline Sensitivity & $98.3 \%$ \\
Specificity & $92.1 \%$ \\
PPV & $95.3 \%$ \\
NPV & $97.2 \%$ \\
Accuracy & $96.0 \%$ \\
\hline
\end{tabular}

\section{DISCUSSION}

Lung cancer is predominantly documented in the right lung and the most frequent sites in decreasing order are: right superior lobe, left superior lobe, right inferior lobe, left inferior lobe and right middle lobe (8)). In this study, maximum lesions were detected on the right upper zone of the lung. Twenty patients had mass in the right upper zone followed by 10 in left upper zone, 11 in right mid zone, 6 in left mid zone and 44 patients had multiple zone involvement. Similar findings were observed by Uddin and Akhtar (1999) (9). Shetty et al (2005) found on CT scan $44 \%$ bronchogenic carcinoma on right lung, $36 \%$ on left lung and $20 \%$ on both lungs(10).

In this series, 39 (37.5\%) patients of bronchial mass lesion had single lesion and $65(62.5 \%)$ had multiple lesions. This finding differs from that of Henschke et al (1999) (5). Among the 233 individuals with a positive CT screening test in their series, 159 (68\%) had single nodule and 43 (18\%) had two nodules. The remaining $14 \%$ had more than two nodules.

In this study, 57 (54.8\%) patients of bronchial mass lesion had speculated mass and $47(45.2 \%)$ patients had smooth lesion. This finding is comparable with Shetty et al (2005) where majority of the patients (85\%) had lesions with speculated margins (10). They found lobulations and smooth margin lesions in in $7 \%$ and $8 \%$ cases, respectively.
CT findings of 104 patients are hilar enlargement $53.8 \%$, cavitation $26 \%$, rib destruction $37.5 \%$, elevation of hemi diaphragm $33.7 \%$, mediastinal involvement $14.4 \%$, chest wall invasion $17.3 \%$, air bronchogram $7.7 \%$, pleural effusion $58.7 \%$ and contrast enhancement $59.6 \%$, Bronchial mass lesions were found moderately enhanced in $50(48.1 \%)$ cases whereas only $50(48.1 \%)$ patients of other lesions were mildly enhanced. Intense enhancement was presented by four patients $(3,8 \%)$.

Maximum patients of bronchial mass lesion and other lesions showed heterogeneous enhancement. They were 60 and 10 respectively.

Khan et al (1985) stated that sensitivity of CT for evaluation of mediastinal nodal metastasis was $83 \%$ and the specificity was $90 \%$ (11). In detection of malignant involvement of the mediastinum Rea et al (1981) found CT scan as $80 \%$ sensitive and $76 \%$ specific modality which is just comparable with our study (12). Accuracy of CT scan of chest in detecting malignant hilar and mediastinal involvement by squamous cell carcinoma of the lung was assayed by Frederick et al (2006) (13). Their results, corrected for reader error, were $92 \%$ sensitive, $92 \%$ specific, and 96\% accurate in the hilum and $95 \%$ sensitive, $77 \%$ specific, and $82 \%$ accurate in the mediastinum involvement. Faling et al (1981) stated that CT scans accurately predicted mediastinal neoplastic lymphadenopathy in 15 or 17 instances of proved mediastinal lymph node metastasis for a sensitivity of $88 \%$; specificity was also high $(94 \%)$ with a truenegative scan in 32 of 34 instances (14).

Several chronic pulmonary diseases are found in association with bronchial carcinoma such as tuberculosis, bronchiectasis, anthracosilicosis, bullous lung disease and pre-existing scar (9). Among 104 patients of the study group the most common diagnosis was malignant lesion in 64 patients (44.2\%). Tuberculosis was diagnosed in 20 patients (19.2\%), inflammatory lesion 14 patients (13.5\%), pneumonic 
consolidation in 4 patients (3.5\%) and fungal lesion 2 patients (1.9\%). Uddin and Akhtar (1999) found twenty patients $(6.60 \%)$ of their study group suffered from respiratory tract infections, 14 (4.62\%) from pulmonary tuberculosis and 3 from asthma (9).

In this study, among 100 patients, cytopathology confirmed malignancy in $62(62 \%)$ cases and other benign conditions in $38(38 \%)$ cases. The most common lung malignancy was squamous cell carcinoma. Twenty nine patients (46.8\%) were diagnosed cytopathologically as squamous cell carcinoma. Adenocarcinoma was diagnosed in 23 patients (37.1\%), small cell carcinoma in 6 patients (9.7\%) and large cell carcinoma in $4(0.7 \%)$ patients. Out of 38 benign condition cytopathology confirmed pulmonary TB in 19 patients (50\%), benign bronchial lesions in 7 patients (18.4\%), nonspecific inflammatory lesions in 8 (21.1\%), and pneumonic consolidation 4 patients (10.5\%). Uddin and Akhtar (1999) found 157 cases $(51.82 \%)$ of squamous cell carcinoma in their series followed by $19.14 \%$ small cell carcinoma, $13.86 \%$ adenocarcinoma, $7.26 \%$ large cell carcinoma, $6.6 \%$ undifferentiated carcinoma,

$0.66 \%$ bronchioalveolar carcinoma, $0.33 \%$ adenosquamous carcinoma and $0.33 \%$ malignant fibrous histocytoma (9). Quiyyum et al (2002), Hasanat et al (2006), Shetty et al (2005), Gupta et al (1998) and Quinn et al (1996) showed similar results $(10,15-18)$.

In this study, the sensitivity, specificity, PPV, NPV and accuracy of CT to diagnose bronchogenic carcinoma was $98.3 \%, 92.1 \%, 95.3 \%, 97.2 \%$ and $96.0 \%$, respectively (19). Colice et al (1985) found that CT was a moderately accurate modality in predicting the presence of air way abnormalities with sensitivity from 63 to $85 \%$ and specificity from $61-77 \%$. The overall sensitivity and specificity of CT scan in the detection of tracheobronchial malignancies were 59\% and $85 \%$ as stated by Finkelstein et al (2003) (20).

\section{CONCLUSION}

CT findings of the present study correlated well in most of the cases with the cytopathological results. It can therefore be concluded that CT scan is useful modality in the evaluation of hilar and mediastinal involvement in bronchogenic carcinoma.

\section{REFERENCES}

1. Hecht SS. Tobacco Smoke Carcinogens and Lung Cancer: Review. J Natl Cancer Inst 1999;91:1194-1210.

2. Hashmi FI, Mpe MJ, Bronchogenic carcinoma: Has the outlook changed? SA Fam Pract 2004;46(8): 26-27.

3. Heitzman ER. The Role of Computed Tomography in the Diagnosis and Management of Lung Cancer: An Overview. Chest 1986;89(4): 237S-241S.

4. Remy-Jardin M, Remy J, Giraud F, Marquette CH. Pulmonary nodules: detection with thick-section spiral CT versus conventional CT. Radiology 1993; 187:513-20.

5. Henschke CI, McCauley DI, Yankelevitz DF. Early Lung Cancer Action Project: overall design and findings from baseline screening. Lancet 1999;354:99-105.

6. MacMahon H, Engelmann R, Behlen FM. Computer-aided diagnosis of pulmonary nodules: results of a large-scale observer test. Radiology 1999; 213:723-6.

7. Knapp RG and Miller MC. Clinical Epidemiology and Biostatisitcs. Williums \& Wilkins 1992.

8. Della GR, Cuna JR, Santorio A, Pellegrini A. Cancer of lung and pleura. In: Bonadonna G, Guna GR (eds). Hand Book of Medicine Oncology. 3rd edn. Masson spA. Milano 1988, pp. 499-520.

9. Uddin MM, Akhtar PS. Clinicopathological study of bronchogenic carcinoma-A study of 303 cases. J Dhaka Med. Coll. 1999;8(1):28-33.

10. Shetty CM., Lakhkar BN, Gangadhar VSS, Ramachandran NR. Changing pattern of bronchogenic carcinoma: A statistical variation or a reality. Ind J Radiol Imag 2005;15(1):233-238.

11. Khan A, Gersten KC, Garvey J, Khan FA, Steinberg H, Oblique Hilar Tomography, Computed Tomography, and Mediastinoscopy for Prethoracotomy Staging of Bronchogenic Carcinoma. Radiology 1985; 156:295-298.

12. Rea HH, Shevland JE, House AJ. Accuracy of computed tomographic scanning in assessment of the mediastinum in bronchial carcinoma. J Thorac Cardiovasc Surg 1981; 81:825-829.

13. Frederick HM, Bernardino ME, Baron M, Colvin R, Mansour $\mathrm{K}$, Miller J, et al. Accuracy of chest computerized tomography in detecting malignant hilar and mediastinal involvement by squamous cell carcinoma of the lung. Cancer 
2006;54(11):2390-2395.

14. Faling U, Pugatch RD. Jung-Legg Y, et al. Computed tomographic scanning of the mediastinum in the staging of bronchogenic carcinoma. Am Rev Respir Dis 1981; 124: 690-695.

15. Quiyyum MA, Hiron MM, Islam MS, Rouf MA, Rahman SMS, Jahan R. Role of bronchial brushing and bronchial biopsy in the diagnosis of lung cancer. Chest \& Heart Journal 2002;26(2):54-61.

16. Hasanat MA, Ahmed S, Rouf MA, Hiron MM, Rahman MM. Study on histological patterns and smoking habits of lung cancer patients. Chest \& Heart Journal 2006;30(1):47-62.

17. Gupta RC, Purohit SD, Sharma MP, Bhardwaj S. Primary bronchogenic carcinoma: clinical profile of 279 cases from midwest Rajasthan. Indian Chest Allied Sciences 1998; 40: 109-116.

18. Quinn D, Gianlupi A, Broste S. The changing radiographic presentation of bronchogenic carcinoma with reference to cell types. Chest 1996;110: 1474

19. Colice GL, Chappel GJ, Frenchman SM, Solomon DA. Comparison of computerized tomography with fiberoptic bronchoscopy in identifying endobronchial abnormalities in patients with known or suspected lung cancer. Am Rev Respir Dis 1985:131:397-400.

20. Finkelstein SE, Schrump DS, Nguyen DM, Hewitt SM, TF Kunst, Summers RM, Comparative Evaluation of Super High-Resolution CT Scan and Virtual Bronchoscopy for the Detection of Tracheobronchial Malignancies. Chest 2003;124:1834-1840. 